\title{
Initial Results on an MMSE Precoding and Equalisation Approach to MIMO PLC Channels
}

\author{
Stephan Weiss*, Nicola Moret ${ }^{\dagger}$, Andrew P. Millar*, Andrea Tonello ${ }^{\dagger}$ and Robert W. Stewart* \\ ${ }^{*}$ Dept of EEE, University of Strathclyde, Glasgow G1 1XW, Scotland, UK \\ \{stephan,bob\}@eee.strath.ac.uk \\ ${ }^{\dagger}$ Dipartimento di Ingegneria Elettrica Gestionale Meccanica, Universitá di Udine, Udine, Italy \\ \{nicola.moret, tonello\}@uniud.it
}

\begin{abstract}
This paper addresses some initial experiments using polynomial matrix decompositions to construct MMSE precoders and equalisers for MIMO power line communications (PLC) channels. The proposed scheme is based on a Wiener formulation based on polynomial matrices, and recent results to design and implement such systems with polynomial matrix tools. Applied to the MIMO PLC channel, the strong spectral dynamics of the PLC system together with the long impulse responses contained in the MIMO system result in problems, such that diagonlisation and spectral majorisation is mostly achieved in bands of high energy, while low-energy bands can resist any diagonalisation efforts. We introduce the subband approach in order to deal with this problem. A representative example using a simulated MIMO PLC channel is presented.
\end{abstract}

\section{INTRODUCTION}

Many transceiver techniques such as OFDM or optimal filter bank based systems perform block processing [1], [2], whereby degrees of freedom are invested into a guard interval that enables to suppress inter-block interference (IBI). The remaining design can then utilise elegant linear algebraic techniques to achieve optimality in various senses, such as by employing a singular value decomposition of the resulting channel matrix. By applying IBI cancellation first rather than trading it off against various other system errors, error terms are not balanced. Recent systems considering this problem include e.g. [3], [4].

In this paper, we consider a minimum mean square error (MMSE) approach for filter bank design of precoding and equalisation targetting both inter-symbol interference (ISI) and structured noise that has been suggested in [5]. While [5] chooses an elegant polynomial matrix formulation, the lack of tools to address the resulting design problem have led to significant simplifications. Here, we explore the utilisation of a polynomial eigenvalue decomposition in [6], [7], which limits the precoder to a paraunitary design. The paraunitarity will be shown to have beneficial consequences, such as simple power control by well-known waterfilling algorithms [8], as well as the application of inversion techniques for polynomial matrices, which need to be solved for the precoder and equaliser design according to the Wiener approach in [5], [9].

The precoder and equalisation design is based on a formulation by Mertins [5], which is stated in Sec. III. In Sec. IV, some thoughts are provided on the implementation of this design. Inital results of this approach are discussed in Sec. V with an application to simulated MIMO power line channel. Finally, conclusions are drawn in Sec. VI.

\section{A. Notation}

Below, boldface uppercase variables such as $\mathbf{H}$ will indicate matrices, while boldface lowercase or underlined letters represent vector valued variables, such as $\mathbf{v}$ or $\underline{V}$. The operator $\{\cdot\}^{\mathrm{H}}$ indicates Hermitian transpose. For polynomial matrices, such as $\mathbf{H}(z)=\sum_{n} \mathbf{H}[n] z^{-n}$, the parahermitian operator $\{\tilde{\cdot}\}$ implies Hermitian transpose of all matrices and time reversal, i.e. $\tilde{\mathbf{H}}(z)=\mathbf{H}^{\mathrm{H}}\left(z^{-1}\right)=\sum_{n} \mathbf{H}^{\mathrm{H}}[n] z^{n}$. For abbreviation, transform pairs are denoted $\mathbf{H}(z) \bullet-\circ \mathbf{H}[n]$. The z-transform is here used for notational purposes only; no actual transformation is carried out, and all calculations will be performed in the time domain.

\section{System Model}

We assume a PLC channel utilising $M$ wires for transmission - e.g. phase, neutral, and earth in a single-phase system - with some degree of cross-coupling, such that an $M \times M$ MIMO transmission system $\mathbf{C}[n]$ arises, whereby

$\mathbf{C}[n]=\left[\begin{array}{cccc}c_{0,0}[n] & c_{0,1}[n] & \ldots & c_{0, M-1}[n] \\ c_{1,0}[n] & c_{1,1}[n] & & \vdots \\ \vdots & & \ddots & \vdots \\ c_{(M-1), 0}[n] & c_{(M-1), 1}[n] & \ldots & c_{(M-1),(M-1)}[n]\end{array}\right]$,

with $c_{i, j}[n]$ being the channel impulse response between the $j$ th input and the $i$ th output of the system. Additionally, we consider multiplexing $P$ subchannels across the MIMO link $\mathbf{C}[n]$, which in term of notation can be represented by demultiplexing the channel $\mathbf{C}[n]$ into a $M P \times M P$ matrix $\mathbf{H}[n]$. The structure of this channel matrix can be expressed in the z-domain based on $\mathbf{C}(z) \bullet-\circ \mathbf{C}[n]$ by a block-pseudocirculant polyphase description $\mathbf{H}(z) \bullet-\circ \mathbf{H}[n]$

$\mathbf{H}(z)=\left[\begin{array}{cccc}\mathbf{C}_{0}(z) & z^{-1} \mathbf{C}_{P-1}(z) & \cdots & z^{-1} \mathbf{C}_{1}(z) \\ \vdots & \ddots & \ddots & \vdots \\ \mathbf{C}_{P-2}(z) & & \mathbf{C}_{0}(z) & z^{-1} \mathbf{C}_{P-1}(z) \\ \mathbf{C}_{P-1}(z) & \mathbf{C}_{P-2}(z) & \cdots & \mathbf{C}_{0}(z)\end{array}\right]$

where $\mathbf{C}_{p}(z)=\sum_{n} \mathbf{C}[n P+p] z^{-n}$. 


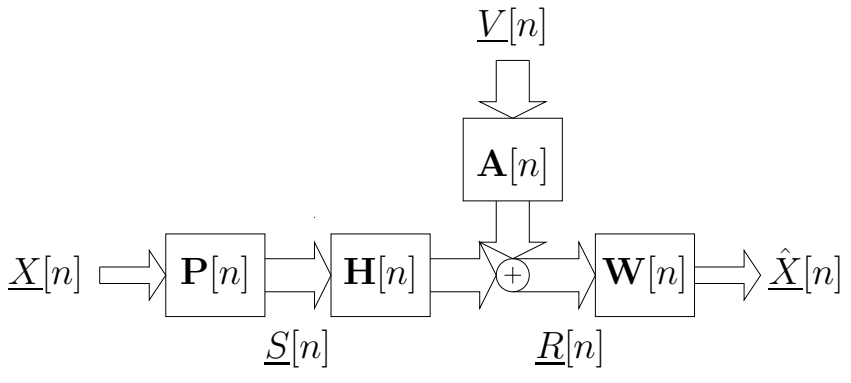

Fig. 1. System model with channel polyphase matrix $\mathbf{H}(z)$ and noise source model $\mathbf{A}(z)$; the transceiver design comprises of a precoder $\mathbf{P}(z)$ and an equaliser $\mathbf{W}(z)$.

Besides co-channel interference (CCI) and ISI caused by $\mathbf{H}(z)$, the received signal is affected by additive noise. Similar to the channel, the noise can be demultiplexed into $P$ subchannels. If the noise is Gaussian and broadband, then a source model or innovations filter matrix $\mathbf{A}(z) \in \mathbb{C}^{M P \times K}$ can linearly relate the $M P$ noise signals corrupting the receive signal to $K$ uncorrelated, mutually independent and identically distributed Gaussian processes with unit variance [10], which are denoted by $\underline{V}(z) \in \mathbb{C}^{K} \bullet-\circ \mathbf{v}[m]$ in Fig. 1. Therefore, the noise power spectral matrix $\mathbf{R}_{w}(z) \in \mathbb{C}^{K \times K}(z)$ as seen at the receiver becomes

$$
\mathbf{R}_{v}(z)=\mathbf{A}(z) \tilde{\mathbf{A}}(z)
$$

As a consequence of (3), $\mathbf{R}_{v}(z)$ is parahermitian, i.e. $\mathbf{R}_{v}(z)=$ $\tilde{\mathbf{R}}_{v}(z)$.

The matrices $\mathbf{P}(z) \in \mathbb{C}^{M P \times N P}(z)$ and $\mathbf{W}(z) \in$ $\mathbb{C}^{N P \times M P}(z)$ describe the precoder and equaliser, respectively. Due to potential oversampling, i.e. $N \leq M$, redundancy is introduced into the transmitted signal $\underline{S}[n]$, which can be exploited to mitigate structured noise and strong modes of the channel transfer function. The design of the linear precoder and equalisation systems $\mathbf{P}(z)$ and $\mathbf{W}(z)$ are the focus of this paper.

\section{MMSE MIMO PRECODING AND EQUALISATION APPROACH}

The precoder and equalisation design is based on a singleinput single-output (SISO) formulation by Mertins [5]. For an arbitrarily selected precoder matrix $\mathbf{P}(z)$, according to a Wiener filter formulation in the z-domain in [5], the MMSE solution for $\mathbf{W}(z)$ can be stated as

$$
\mathbf{W}(z)=\mathbf{R}_{e}(z) \cdot \tilde{\mathbf{P}}(z) \tilde{\mathbf{H}}(z) \mathbf{R}_{v}^{-1}(z),
$$

whereby $\mathbf{R}_{e}(z)$ is given by

$$
\mathbf{R}_{e}(z)=\sigma^{2}\left[\mathbf{I}+\sigma^{2} \tilde{\mathbf{P}}(z) \tilde{\mathbf{H}}(z) \mathbf{R}_{v}^{-1}(z) \mathbf{H}(z) \mathbf{P}(z)\right]^{-1}
$$

with $\sigma^{2}$ being the (equal) power of the signals in $\underline{X}[n]$ in Fig. 1 that feed into the precoder. Assuming that $\mathbf{W}(z)$ has been selected as in (4), the power spectral matrix $\mathbf{R}_{e}(z) \bullet \longrightarrow \mathbf{R}_{e}[\tau]$ defines the MMSE, $\xi_{\mathrm{MMSE}}$, as

$$
\begin{aligned}
\xi_{\mathrm{MMSE}} & =\operatorname{tr}\left\{\mathbf{R}_{e}[0]\right\} \\
& =\operatorname{tr}\left\{\frac{1}{2 \pi} \int_{0}^{2 \pi} \mathbf{R}\left(e^{j \Omega}\right) d \Omega\right\} .
\end{aligned}
$$

With $\mathbf{W}(z)$ selected as the Wiener solution according to (4), the precoder $\mathbf{P}(z)$ can be chosen such that the MSE in (6) is minimised. While in [5], the z-domain notation is chosen for its flexibility, the lack of polynomial matrix tools required a simplification for the solution by creating a non-polynomial precoder $\mathbf{P}_{0}=\mathbf{P}(z)$. This is based on a trick exploited by most block-based transmission systems such as OFDM or optimal filter bank-based precoders and equalisers [1], [2], where the multiplexing factor $P$ is selected larger than the channel order $L$.

With $P>L$, the polyphase components $\mathbf{C}_{p}(z)$ in (2) become zero order, and the channel matrix $\mathbf{H}(z)$ reduces to a first order polynomial, where terms with $z^{-1}$ are restricted to the right upper triangular corner of $\mathbf{H}(z)$ as seen in (2). Using a guard interval, or employing leading or trailing zeros in the transmitter or receiver [1], [2] allows one to extract the zero order component of $\mathbf{H}(z)$. The insertion of a guard interval means that the filter bank is oversampled, and the degrees of freedom associated with the redundancy of this system are utilised to create a zero order transmission matrix thus eliminating IBI.

For the MMSE system in [5] defined by (4) and (5), an implicit selection of $P>L$ leads to a rectangular i.e. oversampled - selection $\mathbf{P}(z)=\mathbf{P}_{0}$ such that the expression $\mathbf{P}_{0}^{\mathrm{H}} \tilde{\mathbf{H}}(z) \mathbf{R}_{v}^{-1}(z) \mathbf{H}(z) \mathbf{P}_{0}$ turns into a non-polynomial formulation.

\section{InVERSION OF PARAHERMITIAN MATRICES}

The work on an eigenvalue decomposition for polynomial matrices in [7] has stimulated a number of tools for polynomial matrix algebra such as the inversion of parahermitian matrices [11] required in (4) and (5), which can address the above MMSE formulation for precoder and equaliser more directy. The required tools are addressed below.

\section{A. Polynomial Eigenvalue Decomposition}

A polynomial eigenvalue decomposition of a parahermitian matrix $\mathbf{R}(z) \in \mathbb{C}^{M \times M}(z)$ is defined as

$$
\mathbf{R}(z)=\mathbf{Q}(z) \mathbf{\Lambda}(z) \tilde{\mathbf{Q}}(z)
$$

whereby $\mathbf{Q}(z) \in \mathbb{C}^{M \times M}(z)$ is paraunitary, i.e.

$$
\mathbf{Q}(z) \tilde{\mathbf{Q}}(z)=\tilde{\mathbf{Q}}(z) \mathbf{Q}(z)=\mathbf{I}
$$

and $\boldsymbol{\Lambda}(z) \in \mathbb{C}^{M \times M}(z)$ is parahermitian and diagonal with diagonal elements $\Lambda_{i}(z)$ ordered such that the power spectral densities $\Lambda_{i}\left(e^{j \Omega}\right)$ fulfill

$$
\Lambda_{i}\left(e^{j \Omega}\right) \geq \Lambda_{i+1}\left(e^{j \Omega}\right), \quad \forall \Omega, \quad i=0 \ldots(M-2) .
$$

The property (10) is called spectral majorisation. While a practical decomposition algorithm developed in [7] will be 


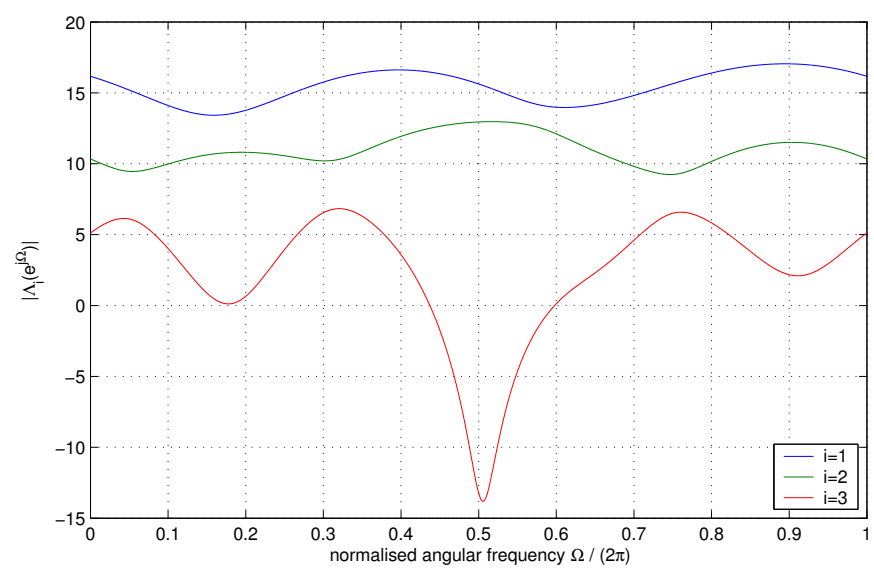

Fig. 2. Power spectra $\Lambda_{i}\left(e^{j \Omega}\right)$ of a spectrally majorised matrix $\mathbf{R}(z)$.

discussed later, an example a the spectrally majorised $\boldsymbol{\Lambda}(z)$ is given in Fig. 2.

\section{B. Polynomial Inverse}

Based on the PEVD, the inverse can be formulated

$$
\mathbf{R}^{-1}(z)=\mathbf{Q}(z) \mathbf{\Lambda}^{-1}(z) \tilde{\mathbf{Q}}(z) .
$$

It is straightforward to show that

$$
\mathbf{R}^{-1}(z) \mathbf{R}(z)=\mathbf{R}(z) \mathbf{R}^{-1}(z)=\mathbf{I} .
$$

The paraunitarity of $\mathbf{Q}(z)$ plays a vital role in the simplicity of this inverse. It remains to invert the diagonal polynomial matrix $\Lambda(z)$, which can be achieved by inverting all elements along on the main diagonal,

$$
\Lambda^{-1}(z)=\left[\begin{array}{cccc}
\Lambda_{0}^{-1}(z) & & & \\
& \Lambda_{1}^{-1}(z) & & \\
& & \ddots & \\
& & & \Lambda_{M-1}^{-1}(z)
\end{array}\right]
$$

whereby $\Lambda_{i}(z) \Lambda_{i}^{-1}(z)=1$. Next, a practical decomposition to determine $\mathbf{Q}(z)$ will be reviewed, before methods to invert the on-diagonal elements $\Lambda_{i}(z)$ are discussed in Sec. IV-D.

\section{Sequential Best Rotation Algorithm}

SBR2 is an iterative broadband eigenvalue decomposition technique based on second order statistics only and can be seen as a generalisation of the Jacobi algorithm. The decomposition after $L$ iterations is based on a paraunitary matrix $\mathbf{U}_{L}(z)$,

$$
\mathbf{U}_{L}(z)=\prod_{i=0}^{L} \mathbf{Q}_{i} \boldsymbol{\Gamma}_{i}(z)
$$

whereby $\mathbf{Q}_{i}$ is a Jacobi rotation and the matrix $\boldsymbol{\Gamma}_{i}(z)$ a paraunitary matrix of the form

$$
\boldsymbol{\Gamma}_{i}(z)=\mathbf{I}-\mathbf{v}_{i} \mathbf{v}_{i}^{\mathrm{H}}+z^{-\Delta_{i}} \mathbf{v}_{i} \mathbf{v}_{i}^{\mathrm{H}}
$$

with $\mathbf{v}_{i}=\left[\begin{array}{lllllll}0 & \cdots & 0 & 1 & 0 & \cdots & 0\end{array}\right]^{\mathrm{H}}$ containing zeros except for a unit element in the $\delta_{i}$ th position. Thus $\boldsymbol{\Gamma}_{i}(z)$ is an identity matrix with the $\delta_{i}$ th diagonal element replaced by a delay $z^{-\Delta_{i}}$.

At the $i$ th step, SBR2 will eliminate the largest off-diagonal element of the matrix $\mathbf{U}_{i-1}(z) \mathbf{R}(z) \tilde{\mathbf{U}}_{i-1}(z)$, which is defined by the two corresponding sub-channels and by a specific lag index. By delaying the two contributing sub-channels appropriately with respect to each other by selecting the position $\delta_{i}$ and the delay $\Delta_{i}$, the lag value is compensated. Thereafter a Jacobi rotation $\mathbf{Q}_{i}$ can eliminate the targetted element such that the resulting two terms on the main diagonal are ordered in size, leading to a diagonalisation and at the same time accomplishing a spectral majorisation.

SBR2 only achieves an approximate diagonalisation after a finite number of iteration steps when off-diagonal elements are smaller than a threshold $\vartheta$,

$$
\mathbf{R}(z)=\mathbf{Q}(z)(\mathbf{\Lambda}(z)+\mathbf{E}(z)) \tilde{\mathbf{Q}}(z)
$$

with $\boldsymbol{\Lambda}(z)$ diagonal and $\mathbf{E}(z)$ a non-sparse error matrix with $\|\mathbf{E}(z)\|_{\infty} \leq \vartheta$. Here, the infinity norm $\|\mathbf{R}(z)\|_{\infty}$ is defined as returning the largest element across all matrixvalued coefficients of the polynomial $\mathbf{R}(z)$,

$$
\|\mathbf{R}(z)\|_{\infty}=\max _{\nu}\left\|\mathbf{R}_{\nu}\right\|_{\infty} .
$$

An alternative stopping criterion is to define a maximum number of iterations for SBR2 [6], [12].

\section{Inversion of Autocorrelation Sequences}

This section addresses the inversion of on-diagonal elements of $\boldsymbol{\Lambda}(z)$. These elements have the properties of autocorrelation sequences, i.e.

$$
r_{i i}[\tau]=r_{i i}^{*}[-\tau] \circ \longrightarrow R_{i i}(z)=R_{i i}^{*}\left(z^{-1}\right) .
$$

This symmetry can be exploited in the inversion process, since the inverse of a linear phase SISO system must also be a linear phase system and therefore have the same symmetry properties [13]-[15]. From $R_{i i}(z) R_{i i}^{-1}(z)=1$ we deduce $r_{i i}[\tau] * s_{i i}[\tau]=\delta[\tau]$ where $s_{i i}[\tau] \circ \longrightarrow S_{i i}(z)=R_{i i}^{-1}(z)$ is the inverse of the auto-correlation sequence. We here use $\mathbf{S}(z)$ to describe the inverse of $\mathbf{R}(z)$ due to potential truncation errors in the methods described below.

\section{E. Time Domain / MMSE Inversion}

The time domain inversion is based on a convolutional matrix desciption of the convolution of an auto-correlation sequence $r[n]$ and its inverse $s[n]$,

$$
\left[\begin{array}{ccccc}
r[N] & & & & \\
\vdots & \ddots & & & \\
r[-N] & & r[N] & & \\
& \ddots & & \ddots & \\
& & r[-N] & & r[N] \\
& & & \ddots & \vdots \\
& & & & r[-N]
\end{array}\right]\left[\begin{array}{c}
s[-T] \\
\vdots \\
s[0] \\
\vdots \\
s[T]
\end{array}\right]=\left[\begin{array}{c}
0 \\
\vdots \\
0 \\
1 \\
0 \\
\vdots \\
0
\end{array}\right]
$$


or

$$
\mathbf{A s}=\mathbf{d}
$$

with $\mathbf{A} \in \mathbb{C}^{(2 T+2 N+1) \times(2 T+1)}, \mathbf{s} \in \mathbb{C}^{(2 T+1)}$ and $\mathbf{d} \in$ $\mathbb{Z}^{(2 T+2 N+1)}$. A solution can be obtained via the left pseudoinverse,

$$
\mathbf{s}=\left(\mathbf{A}^{\mathrm{H}} \mathbf{A}\right)^{-1} \mathbf{A}^{\mathrm{H}} \mathbf{d}
$$

This solution should have the same symmetry properties as $r[n]$, and any deviation from symmetry must be due to numerical problems in the inversion process. The symmetry error

$$
\epsilon=\left\|\mathbf{s}-\mathbf{J} \mathbf{s}^{*}\right\|_{2}^{2}
$$

should be as small as possible.

A minimum mean square error solution to (19) can be obtained by including the noise-to-signal ratio for regularisation purposes.

1) Inversion with Explicit Symmetry Constraint: An illconditioned A can lead to an asymmetric solution in (19). Hence it is advantageous to enforce symmetry in the setup.

This can be performed by a Lagrangian approach, which solves the constrained optimisation problem

$$
\begin{aligned}
\text { find } & \min _{\mathbf{s}}\|\mathbf{A} \mathbf{s}-\mathbf{d}\|_{2}^{2} \\
\text { subject to } & \mathbf{s}=\mathbf{J s}^{*} .
\end{aligned}
$$

Instead of solving this Lagrangian problem, the next section discusses a direct approach of embedding the constraint into the formulation.

2) Inversion with Implicit Symmetry Constraint: The symmetry condition can be incorporated into the system equation by formulating

$$
\left[\begin{array}{cr}
\operatorname{Re}\{\mathbf{A}\} & -\operatorname{Im}\{\mathbf{A}\} \\
\operatorname{Im}\{\mathbf{A}\} & \operatorname{Re}\{\mathbf{A}\}
\end{array}\right] \cdot\left[\begin{array}{c}
\operatorname{Re}\{\mathbf{s}\} \\
\operatorname{Im}\{\mathbf{s}\}
\end{array}\right]=\left[\begin{array}{c}
\mathbf{d} \\
\underline{0}
\end{array}\right] .
$$

In this, the inverse is implicitly constrained by only defining half the response as

$$
\mathbf{w}=\left[\begin{array}{c}
s[-T] \\
\vdots \\
s[1] \\
\frac{1}{2} s[0]
\end{array}\right]
$$

with

$$
\begin{aligned}
& \operatorname{Re}\{\mathbf{s}\}=\left[\begin{array}{cc}
\mathbf{I}_{T} & \underline{0} \\
\underline{0}^{\mathrm{T}} & 2 \\
\mathbf{J}_{T} & \underline{0}
\end{array}\right] \operatorname{Re}\{\mathbf{w}\}=\mathbf{M}_{1} \operatorname{Re}\{\mathbf{w}\} \\
& \operatorname{Im}\{\mathbf{s}\}=\left[\begin{array}{cc}
\mathbf{I}_{T} & \underline{0} \\
\underline{0}^{\mathrm{T}} & 0 \\
-\overline{\mathbf{J}}_{T} & \underline{0}
\end{array}\right] \operatorname{Im}\{\mathbf{w}\}=\mathbf{M}_{2} \operatorname{Im}\{\mathbf{w}\}
\end{aligned}
$$

to reconstruct the real and imaginary part of the true solution. Therefore the problem formulation becomes

$$
\underbrace{\left[\begin{array}{rr}
\mathbf{M}_{1} \operatorname{Re}\{\mathbf{A}\} & -\mathbf{M}_{2} \operatorname{Im}\{\mathbf{A}\} \\
\mathbf{M}_{2} \operatorname{Im}\{\mathbf{A}\} & \mathbf{M}_{2} \operatorname{Re}\{\mathbf{A}\}
\end{array}\right]}_{\mathbf{A}_{c}} \cdot\left[\begin{array}{l}
\operatorname{Re}\{\mathbf{w}\} \\
\operatorname{Im}\{\mathbf{w}\}
\end{array}\right]=\underbrace{\left[\begin{array}{c}
\mathbf{d} \\
\underline{0}
\end{array}\right]}_{\mathbf{d}_{c}}
$$
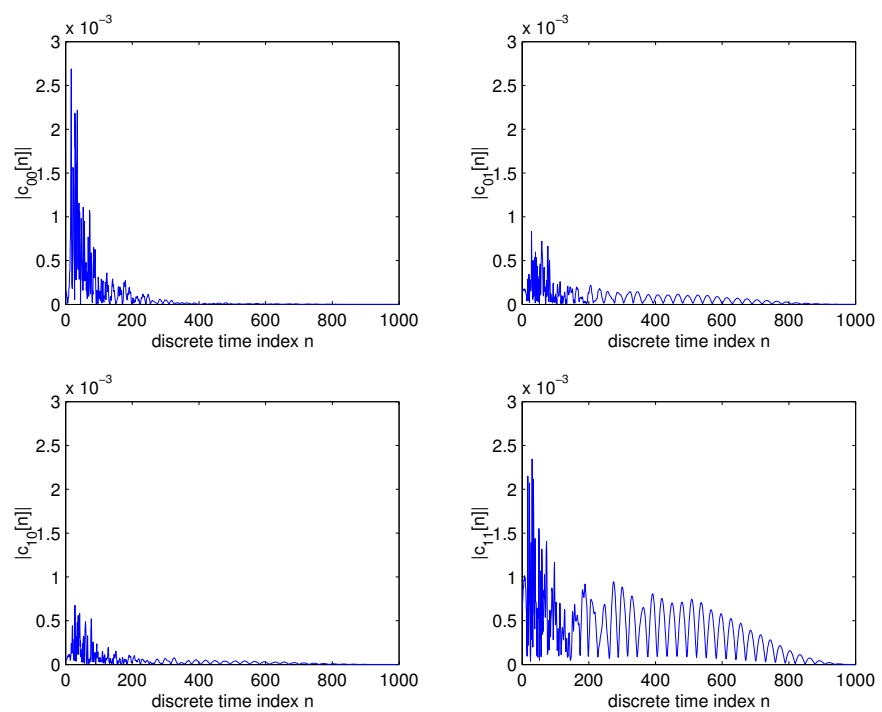

Fig. 3. PLC MIMO channel measurement sample $\mathbf{H}\left(e^{j \Omega}\right)$ over a frequency range of $100 \mathrm{MHz}$

and the solution is reached via the pseudo-inverse

$$
\mathbf{s}=\left[\begin{array}{ll}
\mathbf{M}_{1} & j \mathbf{M}_{2}
\end{array}\right]\left(\mathbf{A}_{c}^{\mathrm{T}} \mathbf{A}_{c}\right)^{-1} \mathbf{A}_{c}^{\mathrm{T}} \mathbf{d}_{c} .
$$

The latter approach has been shown in [11] to be superior in terms of precision and computation complexity to both the unconstrained problem, as well as the formulation involving explicit constraints.

\section{Simulations And Results}

In this section we present some initial results based on a MIMO PLC channel model developed at the University of Udine. This channel model generates channel responses based on a bottom-up PLC channel simulator described in [16]. A representative $2 \times 2$ MIMO channel characterised by the 4 magnitude responses of the constituting SISO subchannels $C_{i j}\left(e^{j \Omega}\right)$ is shown in Fig. 3. The channel is simulated over a bandwidth of $100 \mathrm{MHz}$ and exhibits severe frequency selectivity.

Assuming a much simplified noise model with corruption by additive white Gaussian noise, the noise power spectral matrix is given by a scaled identity matrix, and the denominator of the Wiener solution yields

$$
\mathbf{R}_{e}(z)=\left(\mathbf{I}+\sigma^{2} \tilde{\mathbf{P}}(z) \tilde{\mathbf{H}}(z) \mathbf{H}(z) \mathbf{P}(z)\right)^{-1} .
$$

To minimise the MMSE, the terms in $\tilde{\mathbf{P}}(z) \tilde{\mathbf{H}}(z) \mathbf{H}(z) \mathbf{P}(z)$ need to be maximised, which can be achieved by constructing the precoder matrix $\mathbf{P}(z)$ to support the dominant polynomial eigenmodes of $\tilde{\mathbf{H}}(z) \mathbf{H}(z)$. We first attempt this directly using the $\mathbf{H}(z)$.

\section{A. Direct Approach}

The space-time covariance matrix $\mathbf{R}=\tilde{\mathbf{H}}(z) \mathbf{H}(z)$ in the denominator of the MMSE cost function is characterised in Fig. 4. The decomposition of this matrix is an inportant first step to construct the precoder such that the denominator of 

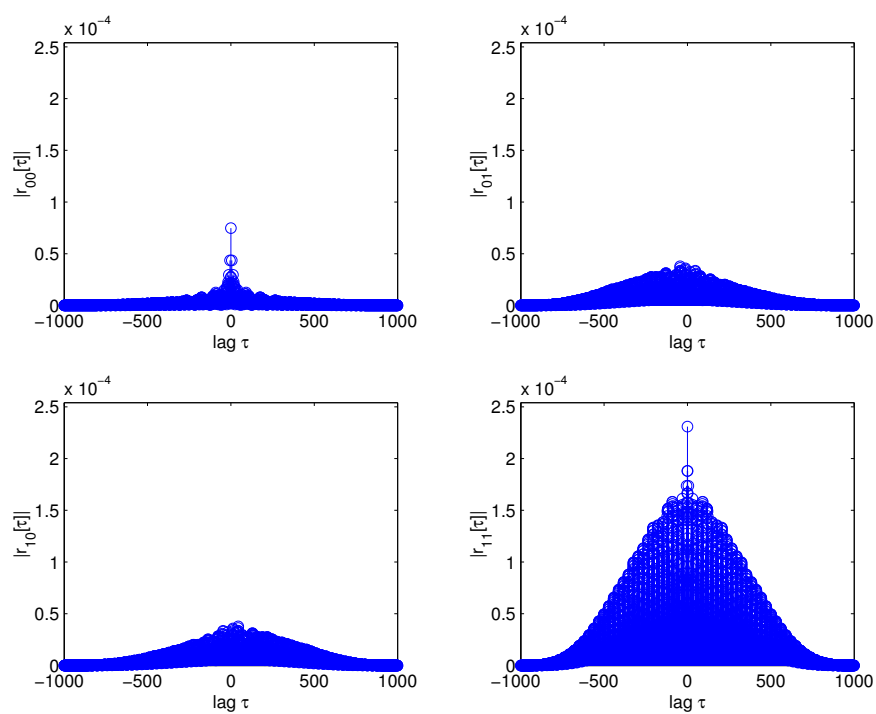

Fig. 4. Space-time covariance matrix $\mathbf{R}=\tilde{\mathbf{H}}(z) \mathbf{H}(z)$,

the MMSE is maximised, e.g. by selecting the strongest polynomial eigenmodes for transmission via $\mathbf{P}(z)$ by extracting the corresponding polynomial eigenvectors from $\mathbf{Q}(z)$ [17]. The advantage of this approach lies in the paraunitarity of the precoder matrix, thus preserving the transmit power, and the fact that the denominator remains an approximately diagonalised matrix, therefore enabling a straightforward inversion according to Sec. IV-D.

While the diagonalisation of the covariance matrix in Fig. 5 appears successful, inspecting the power spectral matrix $R\left(e^{j \Omega}\right)$ in Fig. 6 reveals that the suppression of off-diagonal terms is not great. Also, spectral majorisation is not satisfied across the entire frequency range. The reason can be found in Fig. 7, which shows the low-frequency component of the power spectral matrix, where a large term will dominate the MMSE calculations and therefore obstruct diagonalisation and spectral majorisation in frequency bands where the energy is low. In general, all of the simulated MIMO responses have revealed similar problems due to the very large dynamic range of the channel frequency responses.

The precoder would be designed from the paraunitary polynomial matrix $\mathbf{U}(z)$, such that $\mathbf{R}_{e}(z)$ is diagonal and can be inverted using the method described in Sec. IV-D, thus yielding the solution for the Wiener equaliser matrix $\mathbf{W}(z)$.

\section{B. Subband Approach}

In order to reduce the dynamic range, the MIMO system is combined with a filter bank based transmultiplexer similar to [18], whereby a decomposition into 14 bands oversampled by a factor 16 is achieved using an oversampled filter bank designed according to [11], [19]. The resulting MIMO systems in the 1st and 8th subband are shown in Figs. 8 and 9. Clearly the responses are significantly shorter, thus reducing the numerical complexity of the required polynomial matrix decompositions and inversions.
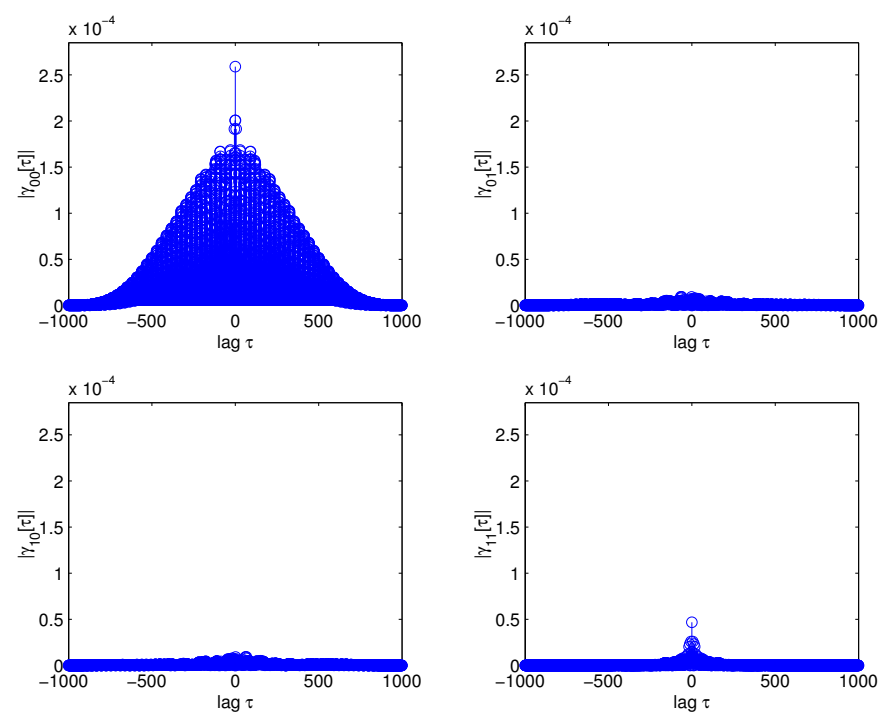

Fig. 5. Space-time covariance matrix in Fig. 4 after approximate diagonalisation by SBR2.
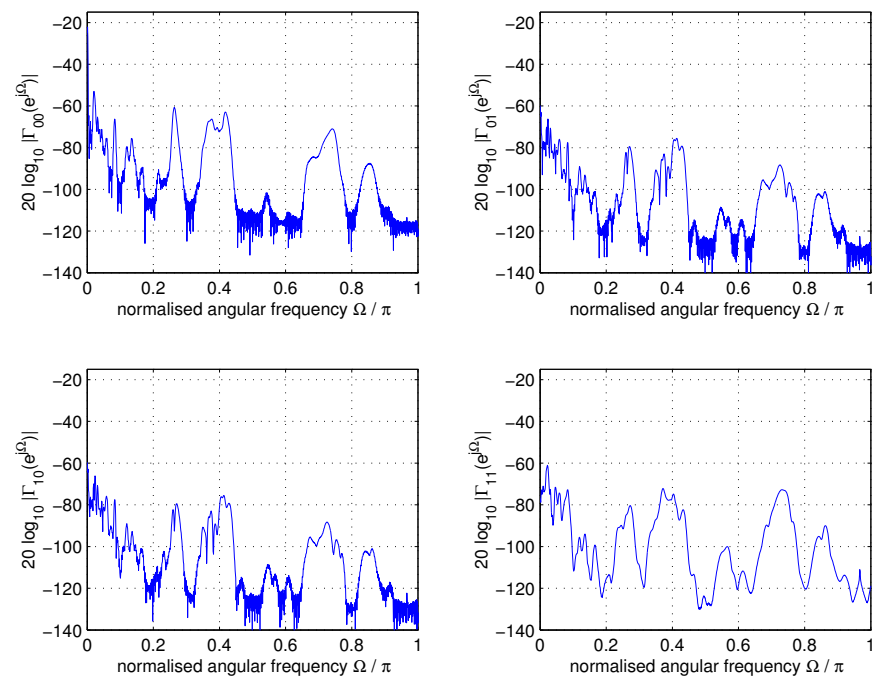

Fig. 6. Power spectral matrix $\boldsymbol{\Gamma}(z)=\tilde{\mathbf{U}}(z) \tilde{\mathbf{H}}(z) \mathbf{H}(z) \mathbf{U}(z)$ after approximate diagonalisation by SBR2.

Fig. 10 depicts the 14 power spectral matrices arising from the transmultiplexer. This can be contrasted against the power spectra after applying SBR2 on the shortened MIMO system in each individual subband, resulting in the systems highlighted in Fig. 11. It is clear that compared to the fullband approach in Fig. 6, the subband approach can further reduce the offdiagonal components even at low gains in the presence of high-energy bands, yielding an improved diagonalisation by SBR2.

The enhanced spectral majorisation of the subband approach is evident from the overlaid power spectra in Fig. 11. With enhanced diagonalisation and spectral majorisation, the denominator term in (5) is also better diagonalised, enabling simple polynomial matrix inversion by means of only inverting the on-diagonal auto-correlation-like term rather than a full-blown 

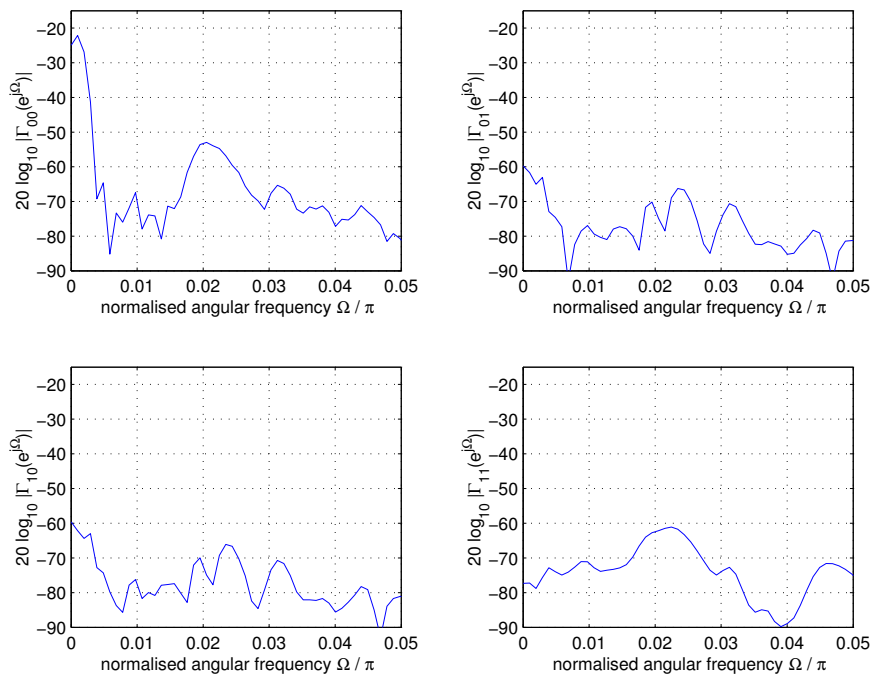

Fig. 7. Low frequency detail of Fig. 6 .
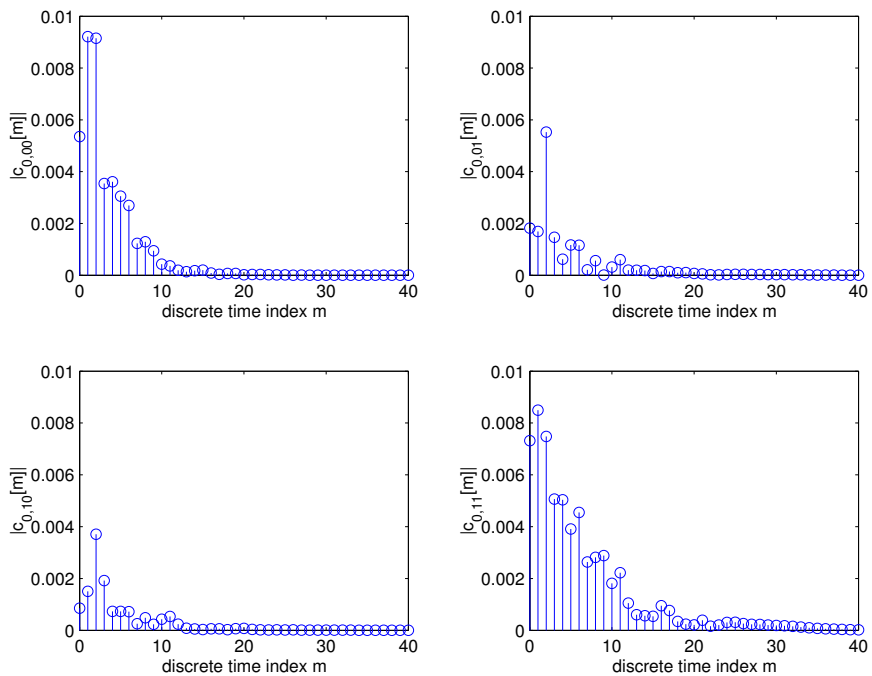

Fig. 8. 1st subband of transmultiplexed MIMO system.

polynomial matrix inversion according the concatenation of methods highlighted in this paper.

\section{CONCLUSiON}

Motivated by MMSE precoding and equalisation without block-processing, we have extended a Wiener filter formulation for polynomial matrices to the MIMO case and explored some polynomial matrix algrebra, in particular the inversion of polynomial matrices based on recent results derived from a polynomial eigenvalue decomposition used for the inversion of parahermitian matrices. The proposed approach has been tested on some simulated PLC MIMO channels.

The diagonalisation achieved in the representative example for a simulated MIMO PLC channel works well in regions with sufficiently high gain in the power spectral density. However, particularly at higher frequencies, which potentially can be gainfully employed for PLC, the diagonalisation is
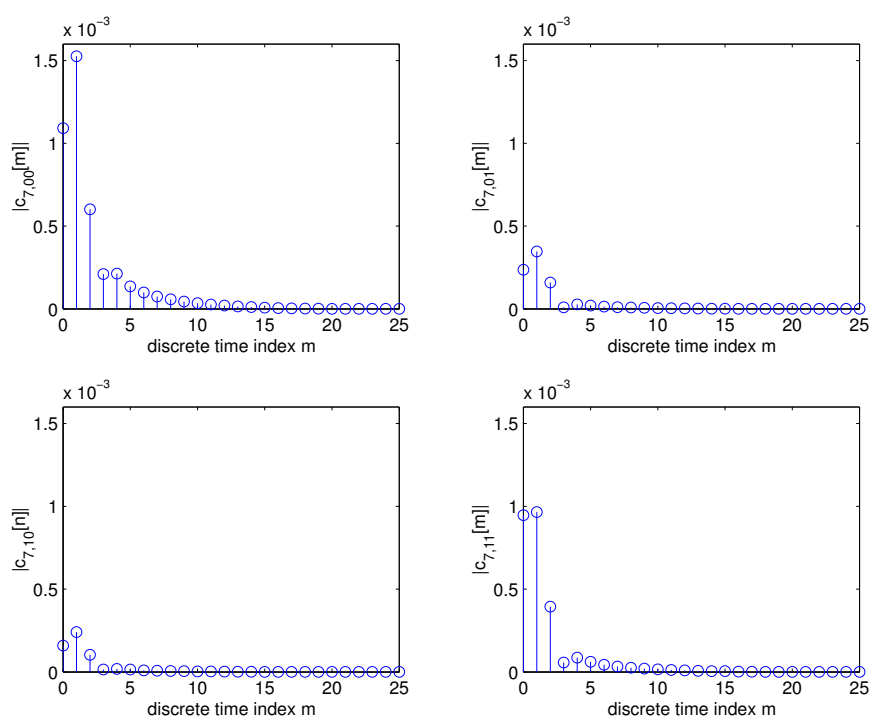

Fig. 9. 8th subband of transmultiplexed MIMO system.
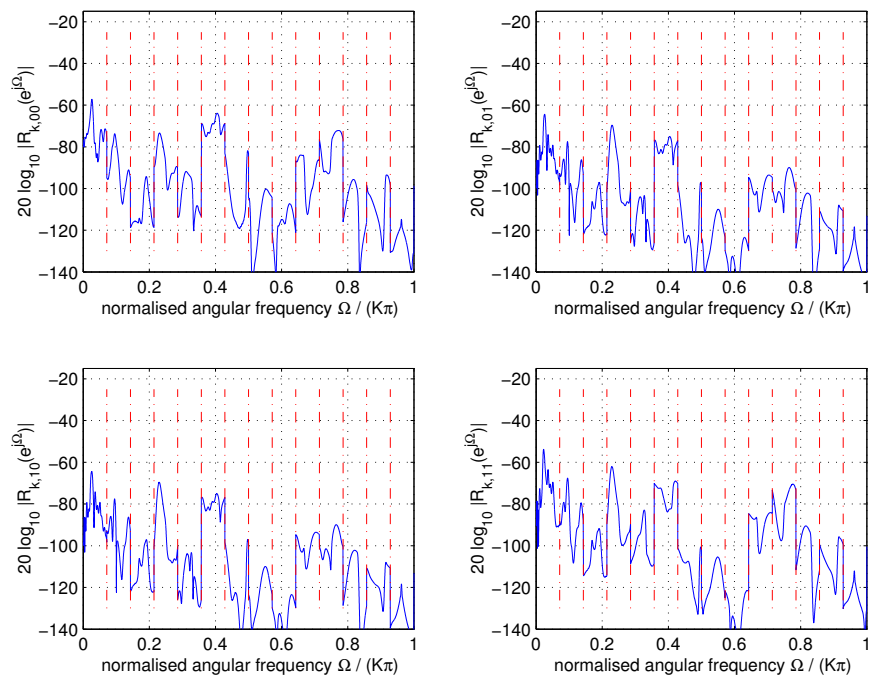

Fig. 10. Concatenated power spectra across the 14 subbands of the transmultiplexer.

insufficient to draw any advantage from the proposed method directly. An improved scheme based on a subband approach has been shown to yield much improved diagonalisation and spectral majorisation, thus enabling the remaining required steps to design and implement a MIMO procoder and equaliser. The subband approach relies on a filter bank transmultiplexer scheme, which divides the channel matrix into frequency bands with reduced dispersion and reduced spectral dynamics. The reduced dispersion results in a significant decrease in the cost of an algebraic polynomial matrix algorithm, while the reduced spectral dynamics yield good diagonalisation over a wide spectral range, thus enabling the inclusion of higher frequency bands for PLC transmission in the proposed precoding and equalisation scheme. 

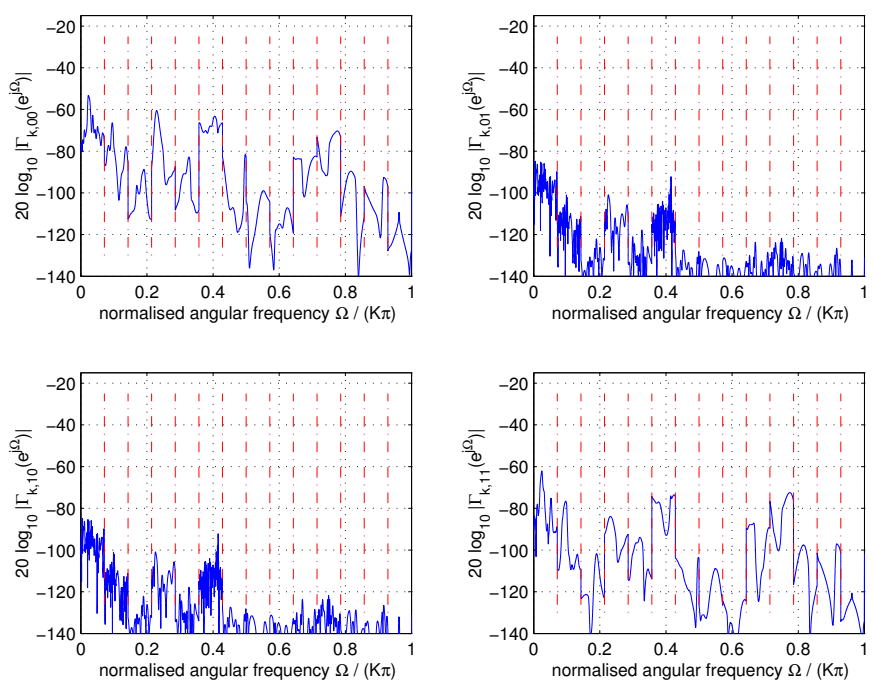

Fig. 11. Concatenated power spectra across the 14 subbands of the transmultiplexed MIMO system diagonalised by SBR2 algorithms operating in every subband.

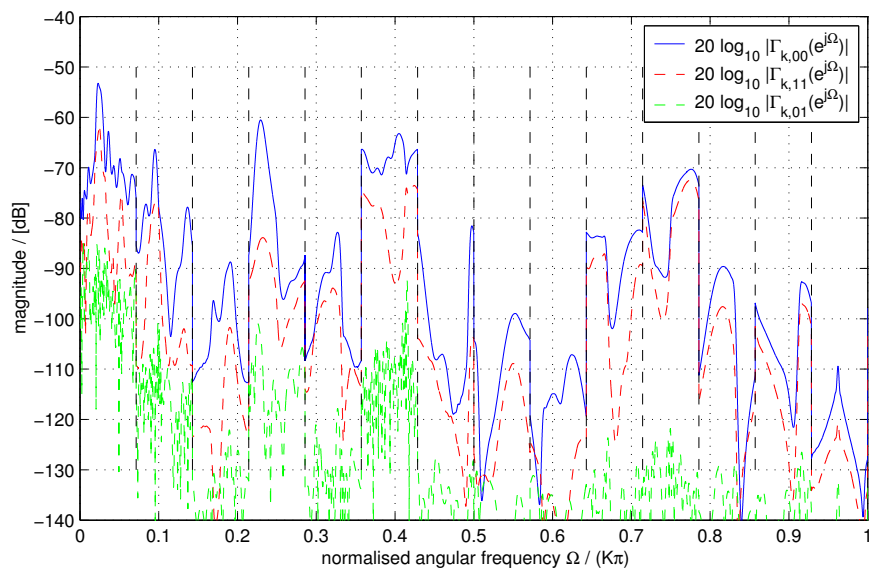

Fig. 12. Overlaid power spectra of Fig. 11, highlighting the diagonalisaion and spectral majorisation properties.

\section{REFERENCES}

[1] A. Scaglione, G. B. Giannakis, and S. Barbarossa, "Redundant Filterbank Precoders and Equalizers. I. Unification and Optimal Designs,' IEEE Transactions on Signal Processing, vol. 47, no. 7, pp. 1988-2006, July 1999

[2] _ , "Redundant Filterbank Precoders and Equalizers. II. Blind Channel Estimation, Synchronization, and Direct Equalization," IEEE Trans actions on Signal Processing, vol. 47, no. 7, pp. 2007-2022, July 1999.

[3] S. D'Alessandro, A. M. Tonello, and L. Lampe, "Bit-loading algorithms for OFDM with adaptive cyclic prefix length in PLC channels," in IEEE International Symposium on Power Line Communications and Its Applications, Dresden, Mar./Apr. 2009, pp. 177-181.

[4] L. Vega and C. Galarza, "Redundancy reduced transceivers and channel identification under impulsive noise," in IEEE International Symposium on Power Line Communications and Its Applications, March 2010, pp. $148-153$.

[5] A. Mertins, "MMSE Design of Redundant FIR Precoders for Arbitrary Channel Lengths," IEEE Transactions on Signal Processing, vol. 51, no. 9, pp. 2402-2409, September 2003.

[6] J. G. McWhirter and P. D. Baxter, "A Novel Technqiue for Broadband SVD," in 12th Annual Workshop on Adaptive Sensor Array Processing, MIT Lincoln Labs, Cambridge, MA, 2004.
[7] J. G. McWhirter, P. D. Baxter, T. Cooper, S. Redif, and J. Foster, "An EVD Algorithm for Para-Hermitian Polynomial Matrices," IEEE Transactions on Signal Processing, vol. 55, no. 5, pp. 2158-2169, May 2007.

[8] D. P. Palomar and J. R. Fonollosa, "Practical Algorithms for a Family of Waterfilling Solutions," IEEE Transactions on Signal Processing, vol. 53, no. 2, pp. 686-695, February 2005.

[9] C. Liu, S. Weiss, S. Redif, T. Cooper, L. Lampe, and J. G. McWhirter, "Channel Coding for Power Line Communication Based on Oversampled Filter Banks," in Proc. International Symposium on Power Line Communications, L. Lampe, Ed., Vancouver, CA, April 2005, pp. 246 249.

[10] A. Papoulis, Probability, Random Variables, and Stochastic Processes, 3rd ed. New York: McGraw-Hill, 1991

[11] S. Weiss, A. Millar, and R. W. Stewart, "Inversion of parahermitian matrices," in European Signal Processing Conference, Aalborg, Denmark, August 2010, pp. 447-451.

[12] S. Redif and T. Cooper, "Paraunitary Filter Bank Design via a Polynomial Singular Value Decomposition," in Proc. IEEE International Conference on Acoustics, Speech, and Signal Processing, vol. 4, Philadelphia, PA, March 2005, pp. 613-616.

[13] A. J. Kanto, "A formula for the inverse autocorrelation function of an autoregressive process," Journal of Time Series Analysis, vol. 8, pp. 311-312, 2008.

[14] W. S. Cleveland, "The inverse autocorrelations of a time series and their applications," Technometics, vol. 14, no. 2, pp. 277-293, May 1972. [Online]. Available: http://www.jstor.org/stable/1267420

[15] D. N. Politis, "Moving average processes and maximum entropy," IEEE Transactions on Information Theory, vol. 38, no. 3, pp. 1174-1177, May 1992.

[16] F. Versolatto, A.M. Tonello, "A MIMO PLC Random Channel Generator and Capacity Analysis," to appear in Proc. International Symposium on Power Line Communications, Udine, Italy, April 2011.

[17] S. Weiss, C. H. Ta, and C. Liu, "A Wiener Filter Approach to the Design of Filter Bank Based Single-Carrier Precoding and Equalisation," in Proc. International Symposium on Power Line Communications, Pisa, Italy, Mar. 2007, pp. 493-498.

[18] N. Moret, A. Tonello, and S. Weiss, "Mimo precoding for filter bank modulation systems based on a polynomial singular value decomposition," in 73rd Vehicular Technology Conference, Budapest, Hungary, May 2011.

[19] M. Harteneck, S. Weiss, and R. Stewart, "Design of Near Perfect Reconstruction Oversampled Filter Banks for Subband Adaptive Filters," IEEE Transactions on Circuits and Systems II: Analog and Digital Signal Processing, vol. 46, no. 8, pp. 1081-1085, 1999. 